\title{
Optically Powered Communication System With Distributed Amplifiers
}

\author{
Xing Xu, Student Member, IEEE, Sigang Yang, Chi Zhang, T. I. Yuk, and Kenneth K. Y. Wong, Senior Member, IEEE
}

\begin{abstract}
An optically powered communication system with distributed amplification is demonstrated using either distributed parametric amplification (DPA) or distributed Raman amplification (DRA) within the dispersion-shifted fiber (DSF) and single-mode fiber (SMF). At the fiber output the residual pump after the distributed amplification is recycled to power the receiver component. Our scheme is also a potential candidate for the 'last mile' transmission. Based on our scheme, 4 channels of $10 \mathrm{~Gb} / \mathrm{s}$ WDM signals are used to obtain practical performance evaluation. In the presence of 10-dB gain for signals, the power penalties of $\leq 1.35-\mathrm{dB}$ at the BER of $10^{-9}$ are achieved for DPA in DSF, and as the comparing counterpart of DPA, counter-pumping DRA induce approximately the same level of power penalty $(\leq 1.55-\mathrm{dB})$ in DSF and $\leq 1.96$-dB in SMF. Co-pumping DRA are also tested in both kind of fibers. Finally, the energy-efficiency issue for different pumping schemes is analyzed.
\end{abstract}

Index Terms-Distributed parametric amplifiers, distributed Raman amplifiers, optical fiber communication, photovoltaic cells, power transmission over fiber, wavelength division multiplexing (WDM).

\section{INTRODUCTION}

$\mathbf{O}$ PTICALLY supplied energy has been well known for a long time, from using solar radiation as power source for satellites in space to the more down-to-earth example of solar battery for household equipments. While in the context of an optically powered communication system, both the optical signal and the power are transmitted in the optical fiber. The advantages of using optical power over traditional electrical means are obvious, such as total immunity to electromagnetic interference, electromagnetic radiation free operation and high electrical resistance which are always concerns in electrical communication systems. And these characteristics also make optical power more environment-friendly than its electrical counterpart.

Running through the short history of optically powered fiber system, Deloach et al. made the first description of an optically powered sound alerter in 1978 [1], soon followed by an optically powered speech communication system [2]. Later in 1989, Kirkham and Johnston [3] reviewed the optically powered devices in the past and presented an optically powered data link. In

Manuscript received May 05, 2010; revised September 03, 2010; accepted September 06, 2010. Date of publication September 13, 2010; date of current version October 13, 2010. This work was supported in part by grants from the research Grants Council of the Hong Kong Special Administrative Region, China under Projects HKU 7179/08E and HKU 7183/09E.

The authors are with the Department of Electrical and Electronic Engineering, The University of Hong Kong, Kowloon, Hong Kong (e-mail: kywong@eee. hku.hk).

Digital Object Identifier 10.1109/JLT.2010.2076346 the following years much of work had been done on how to enhance the power conversion efficiency of the photodiode [4] and analyzing the cost-effective factor of fiber based optically powering system [5]. Specialized networks with optically powered devices were also experimentally evaluated such as fiber-based networks with remote sensor units [5], [6].

When take the 'last mile' transmission into account, optically powered fiber link could easily find its position. Unlike the traditional 'last mile' transmission candidates, i.e., twisted pairs or electrical cable connections, optical fiber has the advantages of high signal-to-noise ratio (SNR), large transmission bandwidth and low attenuation, not to mention the economical factor in the long run of maintenance and repair [7], [8]. Another relatively new choice for the 'last mile' transmission is optical wireless links or free space optics (FSO) [9], [10]. However, in most realistic 'last mile' transmission environments, reflection, refraction or even absorption due to obstacles or unfavorable air conditions like rain or fog, particularly over longer transmission spans, can seriously influence the connections. While in the isolated communication media of optical fiber, there is no such concern. Additionally, in order to secure the SNR for the 'last mile' transmission, distributed amplification is adopted in our scheme. Unlike the discrete amplification which compensates the signal loss between transmission fiber spans, distributed amplification amplifies the signal along the transmission fiber. The most favorable merit of distributed amplification lies in its capability of minimizing the signal power variation along the transmission span which provides the best tradeoff between the noise figure and fiber nonlinearities [11]-[14]. Furthermore, the residual pump after distributed amplification could be recycled to power the receiver component at the fiber output, which guarantees the energy-efficiency of the system.

Recently, with the advances of low-cost high power lasers, low-power-consumption electronic circuits and highly-efficient photodiodes, optically powered networks have attracted wide spread attentions. Optically powered radio-over-fiber remote unit [15], optically powered local-area-network (LAN) systems [16] and optically powered video link [17] have all been demonstrated. The above experiments truly provide potential possibility for the 'last mile' transmission, however, most of these former experiments were multi-mode fiber (MMF) based [15]-[17]. Although MMF could tolerate higher optical power due to its larger core dimension, SMF and DSF could afford much higher data rate and have lower attenuation.

The organization of this paper is as follows. The principles of DPA and DRA are briefly described in Section II. A 4-channel optically powered WDM signal transmission system with distributed amplification is presented in Section III and different 
cases of using either parametric or Raman pump are investigated. The experimental results are given in the first two parts of Section IV. During the experiment the optical-to-electrical power conversion at the receiver is enabled by a commercially available InP-based concentrated photovoltaic (CPV) cell, which can provide a typical power conversion ratio of $20 \%$ at the input optical illumination ranging from $1300 \mathrm{~nm}$ to 1550 $\mathrm{nm}$. Finally, in the last part of Section IV, the energy-efficiency issue of the proposed system is analyzed including discussion and comparison among different pumping cases.

\section{PRINCIPLE}

\section{A. Distributed Parametric Amplification}

DPA, one of the candidates for the distributed signal amplification in long-haul communication links, has been studied in recent research [18], [19]. Although once being regarded as detrimental in some previous research [20]-[23], DPA has several advantages over DRA in real applications including lower pump power, free from double Raleigh scattering (DRS), smaller pump power attenuation and idler generation [18]. Different from the discrete optical parametric amplifier (OPA) in the specially fabricated highly-nonlinear dispersion-shifted fiber (HNL-DSF) [24], [25], DPA adopts the widely available dispersion-shifted fiber (DSF) in which the transmission and amplification of the optical signal can be achieved at the same time [18]. Owe to this important characteristics of DPA, the residual parametric pump can provide power for the remote subscriber of the communication system. Based on the previous work on DPA [18], [19], the emphasis of our experiment is on: (1) the WDM issue; (2) the comparison between DPA and DRA; (3) the detailed energy-efficiency analysis of different distributed amplification schemes.

\section{B. Distributed Raman Amplification}

Raman amplifiers have attracted wide spread research interests in the past decades [26], [27]. Based on Raman effect in the optical fiber and being recognized as the best method to utilize Raman gain, distributed Raman amplification, rather than discrete amplifiers located between fibers, i.e., Erbium-doped fiber amplifier (EDFA) and discrete Raman amplifier, amplifies signals along the transmission fiber itself [28]. The previous study on optically powered system through SMF [29] has achieved simultaneous optical signal and power transmission in a spool of 1-km SMF by using a high power $(22 \mathrm{dBm})$ laser diode with wavelength at $1474 \mathrm{~nm}$. However, the combination of optical power transmission and DRA in longer SMF and DSF has not yet been investigated. In regards to the remaining pump power after DRA, it's promising to use it as the powering source for the receiving component. Thus, as the comparing counterpart of DPA, DRA in both SMF and DSF are investigated thoroughly during the latter part of the experiment. Furthermore, based on the different pumping principles behind DPA and DRA, both co-pumping and counter-pumping schemes are tested for DRA while only co-pumping could be employed in DPA. This favorable merit of DRA enables dual-way amplification for both the down-link and the up-link data in the communication system, while the recycle of residual pump could be fulfilled at both end of the system.

\section{EXPERIMENTAL SETUP}

In order to make practical performance evaluation on our proposed scheme, a 4-channel WDM signal transmission system was introduced. Fig. 1(a) shows the main experimental setup of the system. The input WDM signals, located at the wavelength of $1542.9 \mathrm{~nm}, 1543.76 \mathrm{~nm}, 1544.65 \mathrm{~nm}$ and 1545.45 $\mathrm{nm}$ respectively, were generated by combining the output of four continuous-wave (CW) tunable laser sources (TLS1-4) with three 50/50 couplers and then intensity-modulating them together by the MZM, which was driven by a $10-\mathrm{Gb} / \mathrm{s} 2^{7}-1$ pseudorandom binary sequence (PRBS). The polarization controllers (PC1-4) were used to minimize the insertion loss of each channel by aligning its state-of-polarization (SOP) with the transmission axis of the MZM. A spool of 8-km SMF was deployed to de-correlate different channels in time domain. On the other branch, two different setups were used as the pump: the parametric pump as shown in Fig. 1(b) and the Raman pump as shown in Fig. 1(c) above. The parametric pump, sourced from TLS5 with wavelength at $1548.8 \mathrm{~nm}$, was first phase-dithered by a PM which was driven by a $10-\mathrm{Gb} / \mathrm{s}$ $2^{23}-1$ PRBS, to suppress the stimulated Brillouin scattering (SBS). The insertion loss through PM was minimized using PC6 by aligning the SOP of pump light with the transmission axis of the modulator. A double-stage EDFA scheme was applied to amplify the pump power upto $23 \mathrm{dBm}$. The optical bandpass filter (OBPF2) between two EDFAs was used to reduce the amplified spontaneous emission (ASE) noise level from EDFA2. In order to avoid the reflection and to monitor the SBS level, a circulator (CIR) was inserted after EDFA3. Due to the large output power, instead of using a tunable optical filter, one wavelength-division multiplexing coupler (WDMC3) with 3-nm passing band around $1549 \mathrm{~nm}$ was used to trim down the ASE noise from EDFA3. Different from the parametric pump, the Raman pump was directly generated from a Raman fiber laser with the output power of $26 \mathrm{dBm}$ (with DSF) or 25 $\mathrm{dBm}$ (with SMF) wavelength at $1455 \mathrm{~nm}$. The circulator after the fiber laser was to bypass the reflection and monitor the reflection level. Different cases are described below:

\section{A. Distributed Parametric Amplification in DSF}

Parametric pump (Fig. 1(b)) and DSF (Fig. 1(d)) were adopted. After combining with WDMC1, the parametric pump and the signals were launched together into the DSF which has the length of $10-\mathrm{km}$, nonlinear coefficient $\gamma$ of $2 \mathrm{~W}^{-1} \mathrm{~km}^{-1}$ and the zero-dispersion wavelength $\lambda_{0}$ of $1548 \mathrm{~nm}$. By controlling the variable optical attenuator (VOA1), the signal powers at the input of DSF were maintained around $-19 \mathrm{dBm}$, while the pump power at the same point was $19.5 \mathrm{dBm}$. This ensured that the pump was not depleted and the generation of signal-degraded nonlinear effects, i.e., four-wave-mixing (FWM) and cross-gain modulation (XGM) [30], would be negligible. The SOP of the pump and the signals were aligned by PC5 and PC7 to attain maximum optical parametric gain.

The optical spectra at the output of DSF are shown in Fig. 2. The power of four channels at the input of DSF was 

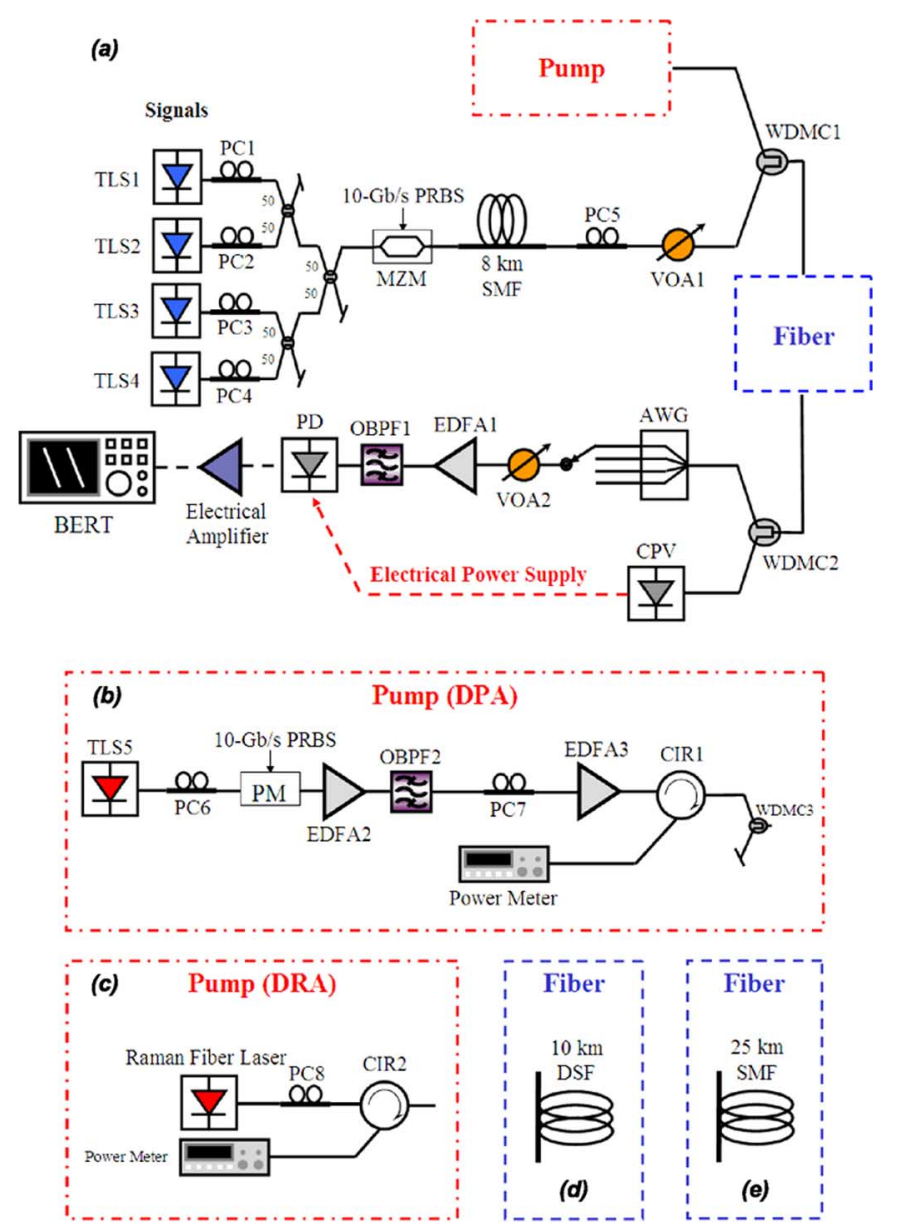

Fig. 1. Experimental setup: (a) main setup for the optically powered WDM signal transmission system with distributed amplification; (b) Setup of the parametric pump for the DPA case; (c) Setup of the Raman pump for the DRA case; (d) 10-km DSF used for both DPA and DRA; (e) 25-km SMF used for DRA. PM: phase modulator, WDMC: WDM coupler, AWG: arrayed-waveguide grating.

$-19.3,-18.92,-19.16$ and $-19.07 \mathrm{dBm}$, respectively. The corresponding on-off parametric gains were 10.06, 10.57, 10.83 and $10.33 \mathrm{~dB}$. At the receiving end, after WDMC3, WDM signals were de-multiplexed by an arrayed-waveguide grating (AWG) and the performance of each channel was then quantified by the bit-error-rate tester (BERT). On the other hand, the residual parametric pump was sent to the CPV cell.

\section{B. Distributed Raman Amplification in DSF}

Raman pump (Fig. 1(c)) and DSF (Fig. 1(d)) were used in the second case. Furthermore, due to the wavelength difference between the parametric pump $(1548.8 \mathrm{~nm})$ and the Raman pump (1455 nm), WDMC1 and WDMC3 were changed into $14 x x / 1530-1580 \mathrm{~nm}$ coupler. For fair comparison with DPA, the signal powers at the input of DSF were maintained unchanged in all three situations, while the Raman pump power at the same point was $23.5 \mathrm{dBm}$.

The optical spectra at the output of DSF with and without Raman pump are shown in Fig. 3. The corresponding on-off Raman gains for each channel were 10.35, 10.72,10.4 and 10.29 $\mathrm{dB}$. The signal receiving part was the same as in the parametric pump case.

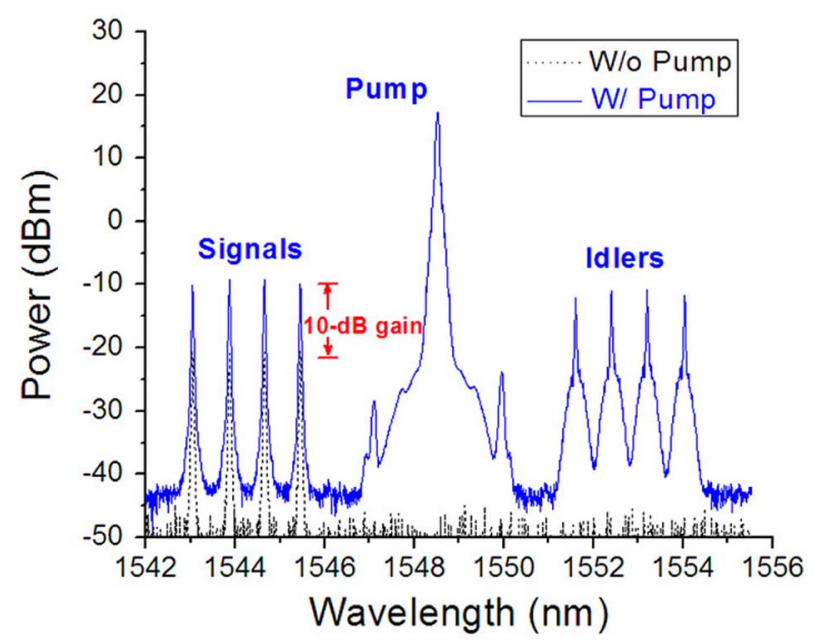

Fig. 2. Optical spectra at the output of DSF with (blue solid line) and without (black dotted line) pump.(OSA resolution bandwidth: $0.02 \mathrm{~nm}$ ).

\section{Distributed Raman Amplification in SMF}

Raman pump (Fig. 1(c)) and SMF (Fig. 1(e)) were used in the third case. Additionally, to compensate the chromatic dispersion induced after passing through the 25-km SMF, one spool of dispersion-compensation fiber (DCF) with a total chromatic dispersion of $-386 \mathrm{ps} / \mathrm{nm}$ was positioned between AWG and WDMC2 in Fig. 1(a). The pump power at the fiber input was $22.5 \mathrm{dBm}$ and the other parts of the setup were maintained the same as in the last case of DRA in DSF. Due to the similarity and to avoid iteration and repetition, the optical spectra of DRA in SMF is not shown here. While the corresponding on-off Raman gains were $10.43,10.78,10.46,10.8 \mathrm{~dB}$.

\section{Counter-Pumping Distributed Raman Amplification}

For the last part of the whole experiment, the counterpumping scheme for DRA was also tested with 10-dB Raman gain in both DSF and SMF, the setup is shown in Fig. 4 above with the WDM signals and the receiver part remaining the same as in Fig. 1(a) while the Ramam pump is shown in Fig. 1(c).

\section{EXPERIMENTAL RESULTS AND DISCUSSION}

\section{A. Distributed Parametric Amplification}

During the experiment, each channel was switched off sequentially to quantify the signal crosstalk amongst channels as shown in Fig. 5, and the spectra were obtained right after the DSF before the WDM coupler and the AWG. It can be observed that there is no observable spurious FWM components (more than 28-dB lower than the signals) generated. The BER plot of each WDM channel was measured and compared against the back-to-back (B2B) results as shown in Fig. 6. Three different configurations were considered during the BER measurement process: (1) only one channel was switched on after DSF without parametric pump; (2) only one channel was switched on after DPA; (3) all four channels were switched on after DPA. Less than $0.28-\mathrm{dB}$ power penalty was introduced for each signal channel when the parametric pump was switched off in the first case. While error-free operations were attained for all channels and the power penalties incurred ranged from 1.08 to $1.33-\mathrm{dB}$ 

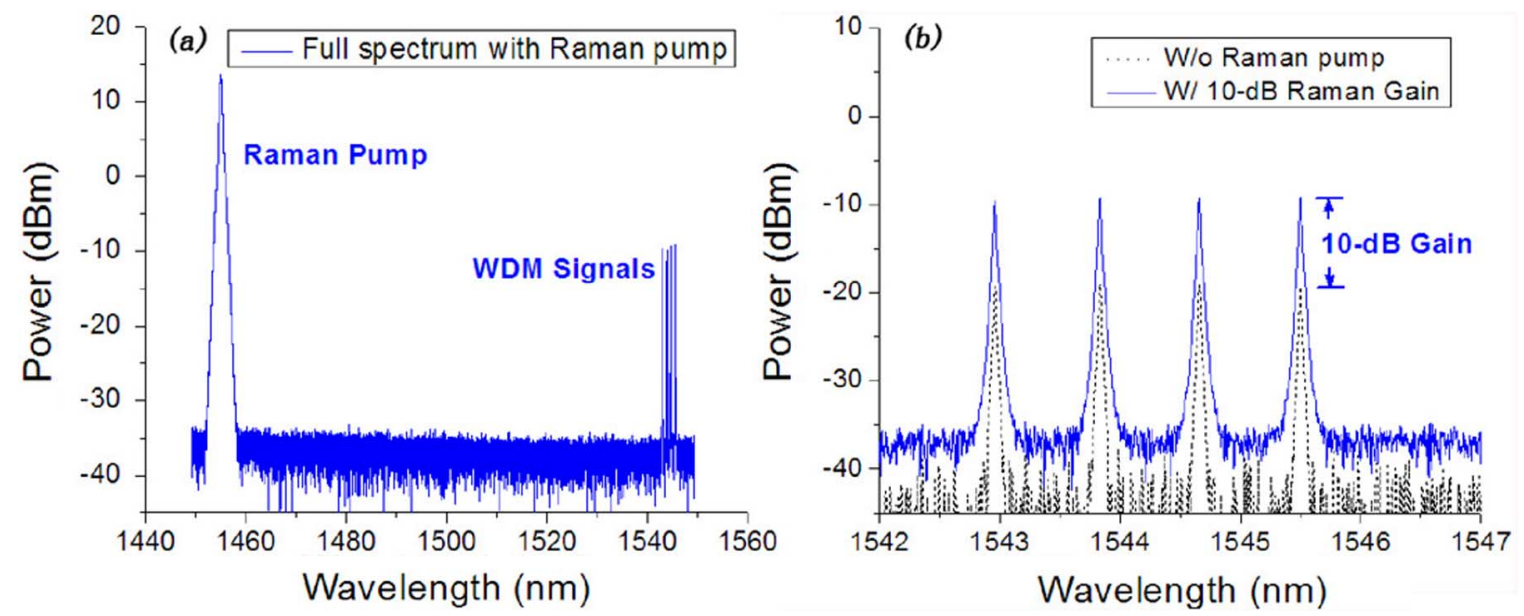

Fig. 3. Optical spectra at the output of DSF with (blue solid line) and without (black dotted line) Raman pump: (a) Full spectrum with 10-dB Raman gain; (b) Enlarged spectra of WDM signals. (OSA resolution bandwidth: $0.02 \mathrm{~nm}$ ).

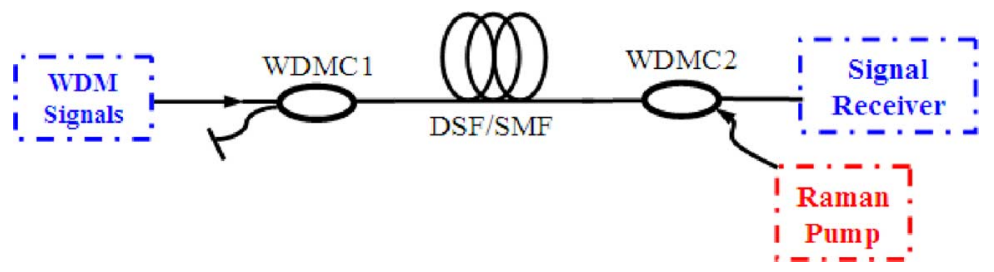

Fig. 4. Experimental setup for the counter-pumping distributed Raman amplification using either DSF or SMF.
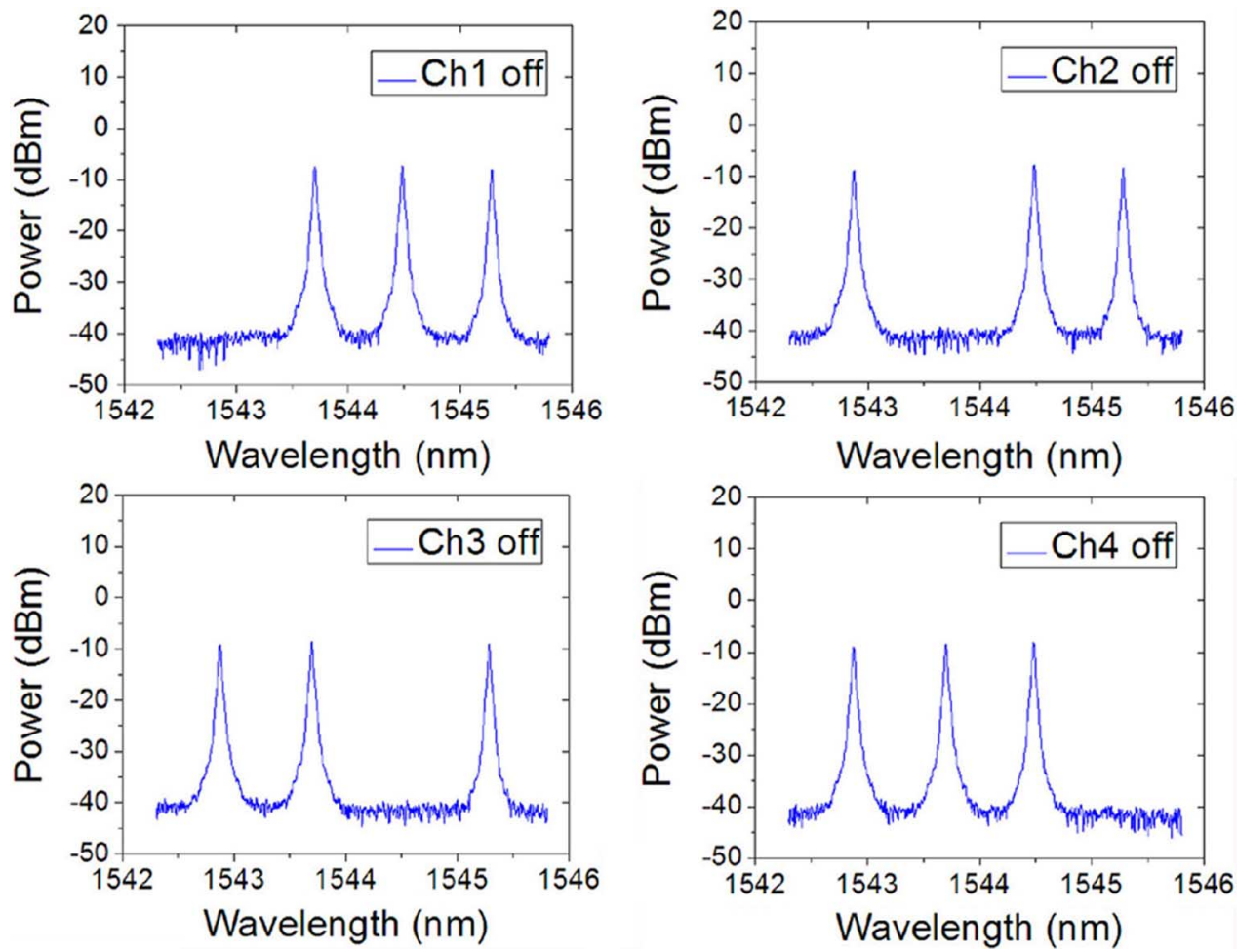

Fig. 5. Spectra with one WDM channel off after DPA. (OSA resolution bandwidth: $0.02 \mathrm{~nm}$ ).

at the BER of $10^{-9}$ for single channel case, additional penalties of less than 0.2-dB were measured in the four channel case which indicate nearly negligible impact on these WDM chan- nels. The insets of Fig. 6 also list the measured eye diagrams of the received signals. Clear and widely open eye diagrams were observed for all four channels. 

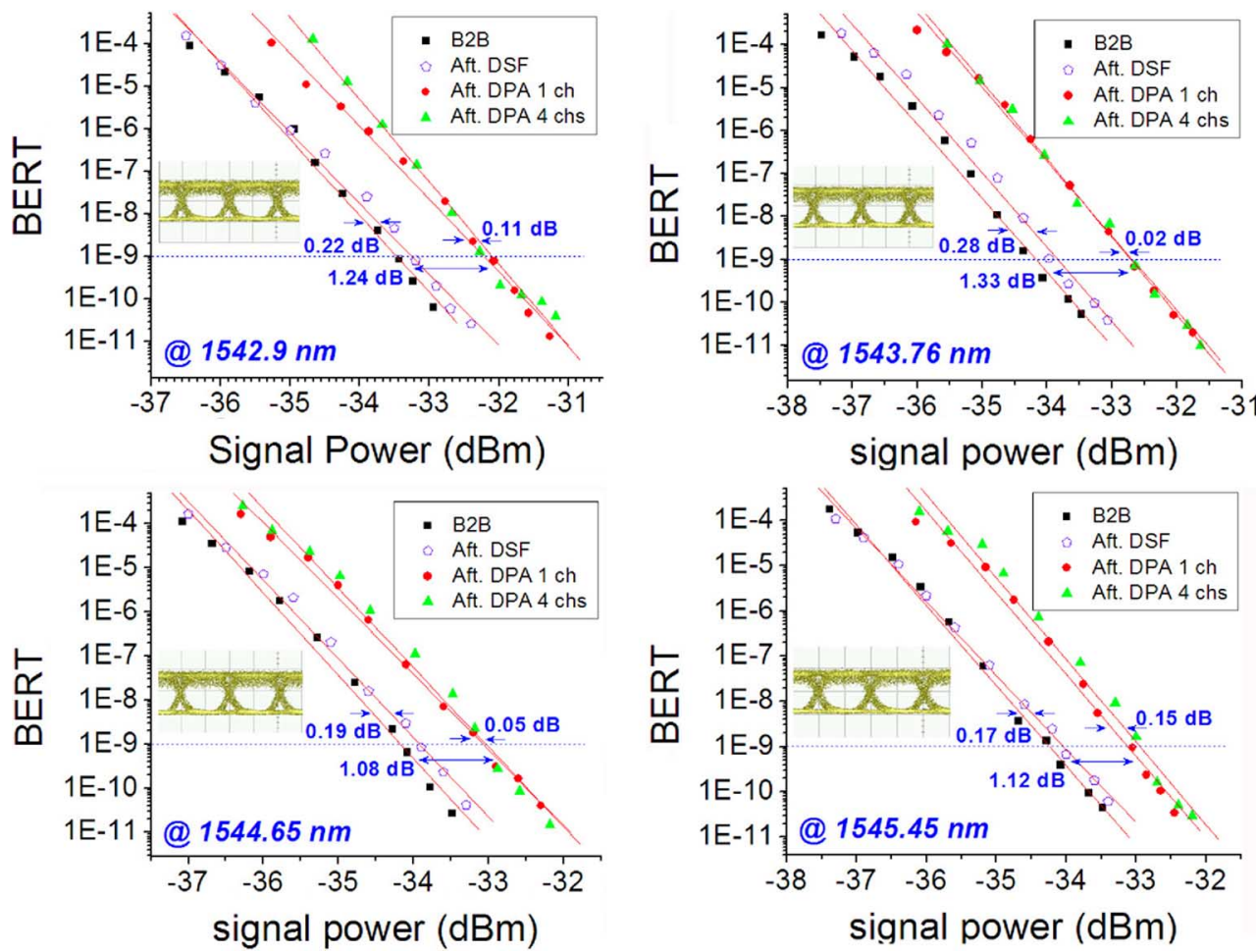

Fig. 6. BER plots after DPA in DSF with the inset of eye diagrams for each channel: back-to-back (square), after DSF without DPA (hollow pentagon), only one channel on with DPA (circle), all 4 channels on with DPA (triangle).

\section{B. Distributed Raman Amplification}

The corresponding BER of each channel in different DRA schemes are shown in Figs. 7 and 8. BER results for co-pumping, counter-pumping and the case without Raman pump for each channel are put in the same figure to make further performance comparison. Less than $0.35-\mathrm{dB}$ power penalty was measured for each channel after passing through the DSF without the pump, while slightly higher penalty (less than 0.69-dB) was obtained for the SMF case. Error-free operations were attained in all situations, the power penalties incurred ranged from 1.3 to $1.55-\mathrm{dB}$ at the BER of $10^{-9}$ for the counter-pumping DRA in DSF and 1.84 to $1.96-\mathrm{dB}$ for the SMF case. Additional penalties of no more than 2.36-dB were measured for both the co-pumping DRA in DSF and SMF. The inset eye diagrams shown in Figs. 7 and 8 list out the measured 10-Gb/s NRZ signals for four different DRA cases: (1) co-pumping DRA in DSF (Fig. 7 upper inset); (2) counter-pumping DRA in DSF (Fig. 7 lower inset); (3) co-pumping DRA in SMF (Fig. 8 upper inset); (4) counter-pumping DRA in SMF (Fig. 8 lower inset).

\section{Discussion and Energy-Efficiency Analysis}

The noise observed on the mark level after DPA was mainly due to the conversion of the phase modulation of the parametric pump to the signal intensity modulation (PM to IM) during the parametric process along the DSF. While for the DRA, it is clear to see from eye diagrams that the signal quality of counter-pumping scheme was better than its co-pumping counterpart at the mark level. The main reason is that the counter-pumping scheme has a better relative intensity noise
(RIN) tolerance than the co-pumping scheme [31]. Additionally, from the BER curves shown in the previous two subsections, for the co-pumping scheme in DSF, the amplification performance of DPA is much better than DRA considering the power penalties induced (DPA: $\leq 1.35-\mathrm{dB}$, DRA: $\leq 3.88-\mathrm{dB}$ ). While the counter-pumping DRA in DSF has nearly the same level of performance comparing to DPA (DPA: $\leq 1.35-\mathrm{dB}$, DRA: $\leq 1.55-\mathrm{dB}$ ). Larger power penalties occurred during both the case of pump-free and counter-pumping DRA in SMF were due to the imperfect matching between the SMF and the DCF used for dispersion compensation (SMF: $425 \mathrm{ps} / \mathrm{nm}$, DCF: $-386 \mathrm{ps} / \mathrm{nm}$ ).

When take the energy-efficiency issue into account, although the attenuation of optical fiber is much smaller when comparing with traditional electrical power transmission line, the loss of optical power is still not negligible especially for long-distance delivery. The power consumption of optical pump during the transmission and the amplification process can be divided into several parts as shown in the equation below

$$
P_{\text {total }}=P_{c}+P_{f}+P_{t}+P_{r}(\mathrm{~dB}) .
$$

With $P_{\text {total }}, P_{c}, P_{f}, P_{t}$ and $P_{r}$ represent the total power consumption, the power loss due to the WDM couplers and circulators, the optical power attenuation in the fiber, the power transferred to signals during the amplification process and the reflected power (including reflection from SBS, Rayleigh scattering and connectors), respectively.

The actual power losses due to different parts were measured during the experiment for each case as shown in Table I below. $P_{p}$ represents the pump power before the circulator and $P_{\mathrm{CPV}}$ 

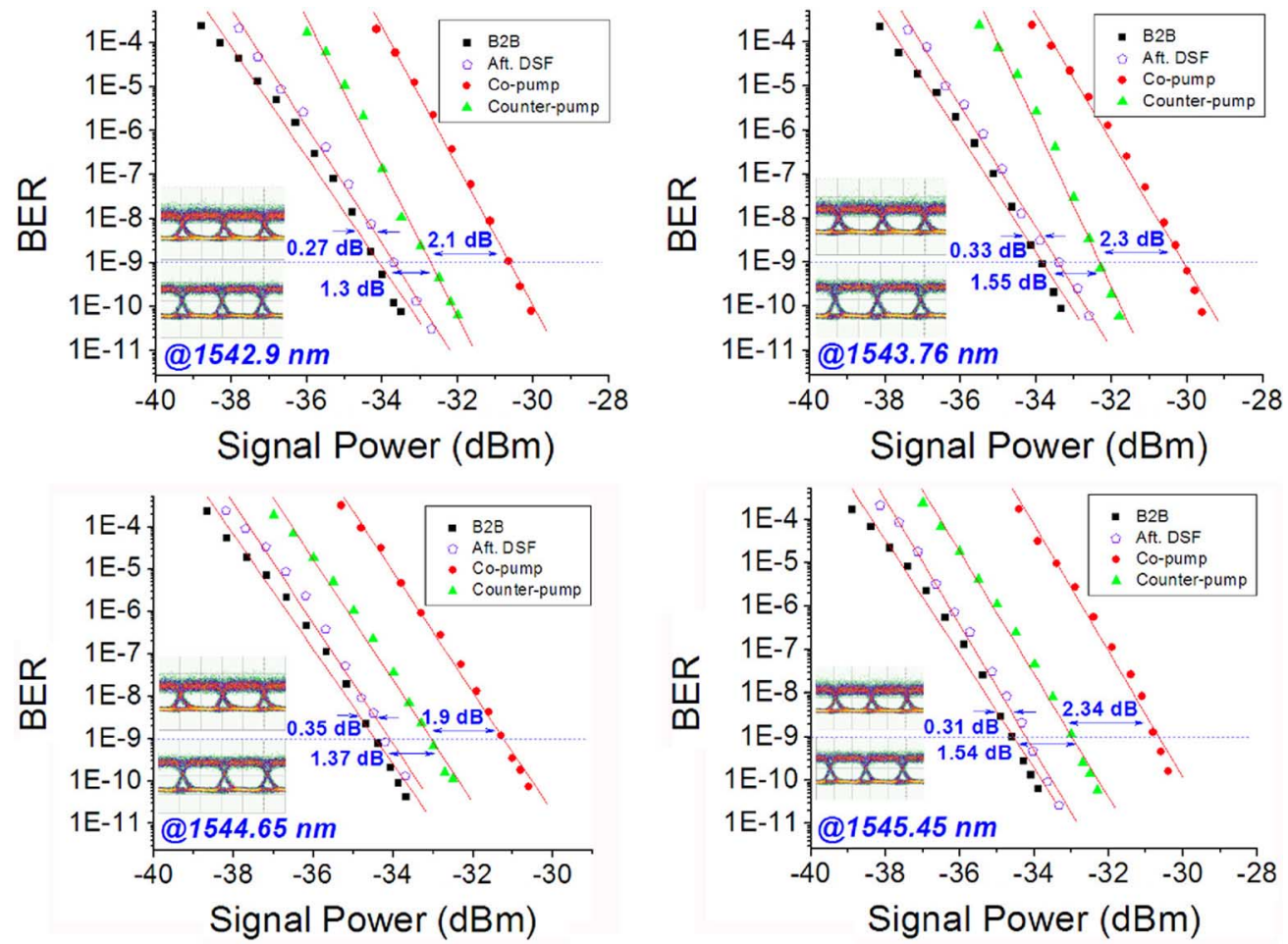

Fig. 7. BER plots after DRA in the 10-km DSF with insets of eye diagrams (upper: co-pumping case; lower: counter-pumping case) for each channel: back-to-back (square), after DSF without DRA (hollow pentagon), co-pumping scheme (circle), counter-pumping scheme (triangle).

stands for the residual pump power at the CPV cell input. Equation (2) shows the relationship between $P_{p}$ and $P_{\mathrm{CPV}}$

$$
\begin{aligned}
P_{p}(\mathrm{dBm}) & =\left(P_{\mathrm{CPV}}+P_{\mathrm{total}}\right)(\mathrm{dBm}) \\
& =\left(P_{\mathrm{CPV}}+P_{c}+P_{f}+P_{t}+P_{r}\right)(\mathrm{dBm})
\end{aligned}
$$

From the table, it could be seen that for the same 10-dB signal gain in DSF, about 3-dB lower power was needed for the parametric pump in DPA than the Raman pump in DRA. On the other hand, the transmitted optical power is about $0.5-\mathrm{dB}$ larger for DPA than for DRA. The main reasons are larger loss for the $1455 \mathrm{~nm}$ Raman pump and the higher reflection power level observed during the DRA experiment. And this make DPA a better choice when DSF is chosen as the transmission media in our scheme. However, DPA still has its limitations [18]: if larger gain bandwidth and flattened gain spectrum are required for WDM system, multi-pump DRA will become the better candidate [28], [32], [33]. Using SMF as the transmission media is another story, as DPA is not feasible for C-band signals in SMF which has the zero-dispersion wavelength located near $1300 \mathrm{~nm}$, only DRA could be applied. But DPA could still be a possible choice for the signal transmission around $1300 \mathrm{~nm}$ in SMF.

Fig. 9 shows the output curve of the CPV cell with different input optical power at pump wavelengths. According to the curves, the corresponding output could reach $4.524 \mathrm{~V}$ for the DPA, $4.505 \mathrm{~V}$ for the DRA in DSF and $4.03 \mathrm{~V}$ for the DRA in SMF. These were large enough for the bias voltage of the $5-\mathrm{V}$ photodiode (PD) used at the receiving end as the optical signal to electrical signal converter in our experiment.
Besides the loss control during the power transportation, to further guarantee the energy-efficiency, another key device is the photovoltaic cell for the power conversion from optical to electrical form. In our experiment, a InP-based concentrated photovoltaic $(\mathrm{CPV})$ cell was used with a typical power conversion ratio $\eta_{c}$ of $20 \%$ (maximum up to $26 \%$ ) at the input optical power of less than or equal to $150 \mathrm{~mW}$, and both the efficiency and output voltage decrease with higher input power. For example, at $1 \mathrm{~W}$ input, it could drop to $10 \%$ efficiency, but the absolute output power $P_{\text {out }}(100 \mathrm{~mW})$ is still higher than $30 \mathrm{~mW}$, when at $20 \%$ efficiency. Actually, specially designed photovoltaic cell could reach much higher $\eta_{c}$ of more than 50\% [34], [35], and such kind of device could further promote the energy-efficiency of our scheme

$$
P_{\text {out }}(\mathrm{mW})=P_{\mathrm{CPV}}(\mathrm{mW}) \times \eta_{c}
$$

According to Table I and (3), the input optical power at CPV cell $P_{\mathrm{CPV}}$ ranging from 10.3 to $15.2 \mathrm{dBm}$ (10.7 to 33.1 $\mathrm{mW}$ ) could generate the output power $P_{\text {out }}$ of 2.14 to $6.6 \mathrm{~mW}$. At the level of several milli-watt mean power consumption, remote-end subscribers including temperature sensors, motion sensors and still picture cameras [17] could be easily powered up by our scheme. Moreover, as analyzed in the preceding paragraph, if photovoltaic cell with higher conversion efficiency used, devices of higher power consumption like electrical amplifiers could be powered up at the receiver end. Thus, besides possible utilization into the 'last mile' transmission, the potential application of our proposed scheme lies in optically powered sensor network located in relative inaccessible environment. Furthermore, our scheme of combining DPA or DRA 

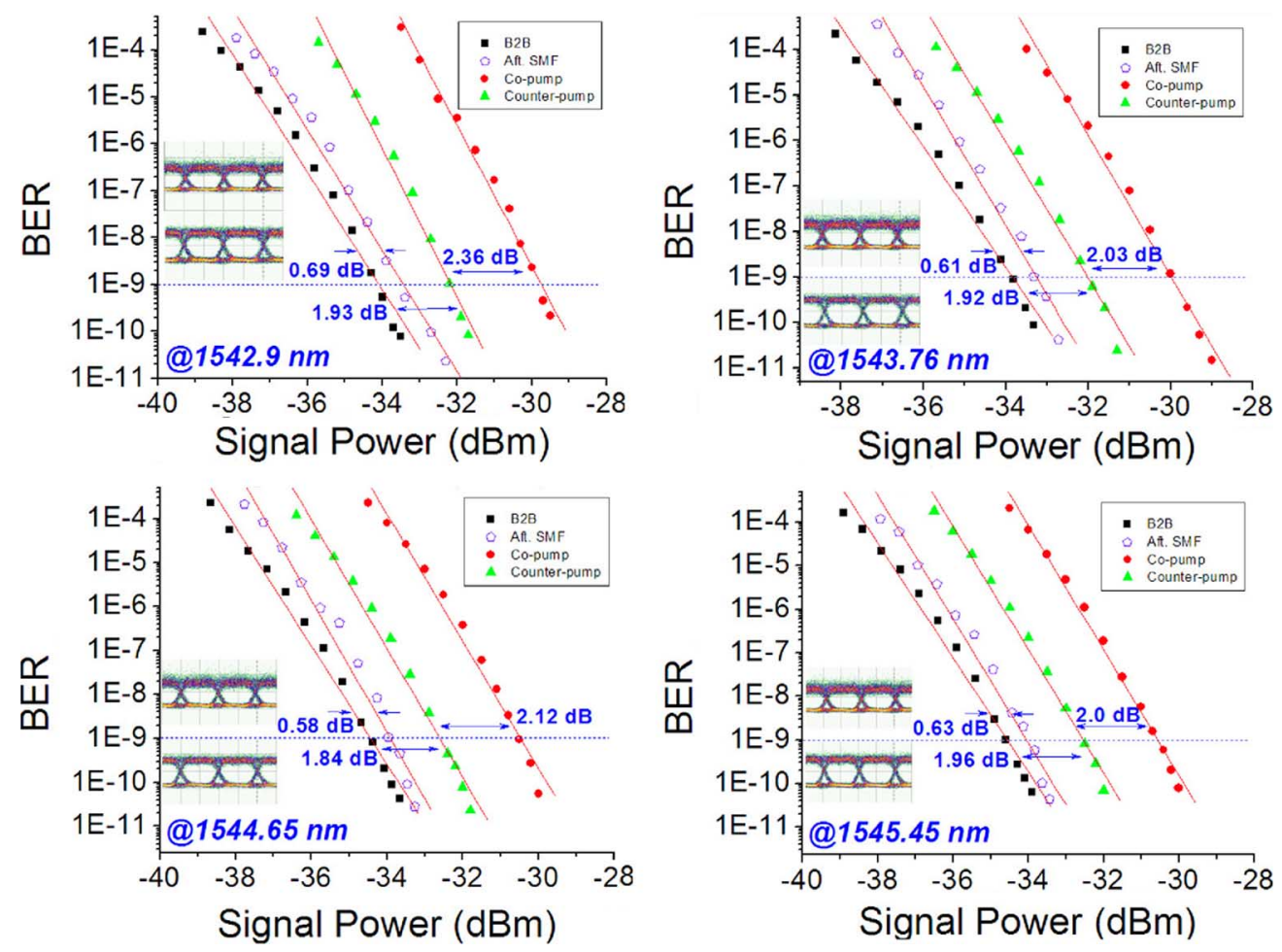

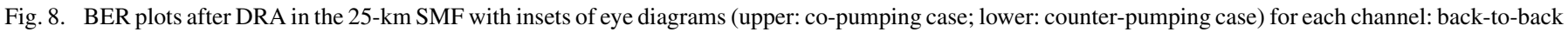
(square), after SMF without DRA (hollow pentagon), co-pumping scheme (circle), counter-pumping scheme (triangle).

TABLE I

POWER CONSUMPTIONS FOR DIFFERENT CASES

\begin{tabular}{lccccc}
\hline & $\begin{array}{c}P_{p} \\
(d B m)\end{array}$ & $\begin{array}{c}P_{c} \\
(d B)\end{array}$ & $\begin{array}{c}P_{f} \\
(d B)\end{array}$ & $\begin{array}{c}P_{t}+P_{r} \\
(d B)\end{array}$ & $\begin{array}{c}P_{C P V} \\
(d B m)\end{array}$ \\
\hline DPA in DSF & 23 & 3.5 & 2.6 & 1.7 & 15.2 \\
DRA in DSF & 26 & 2.5 & 3.5 & 5.3 & 14.7 \\
DRA in SMF & 25 & 2.5 & 7.5 & 4.7 & 10.3 \\
\hline
\end{tabular}

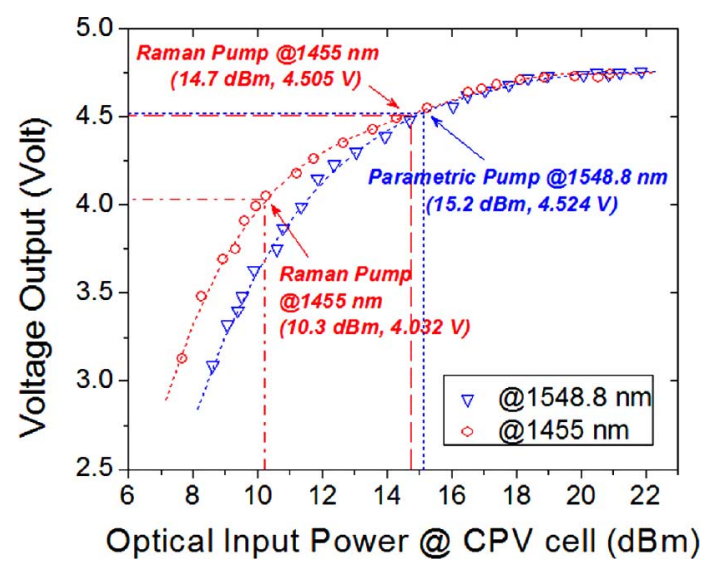

Fig. 9. Response curves of the CPV cell with the input pump light @ $1548.8 \mathrm{~nm}$ (triangle) and @ $1455 \mathrm{~nm}$ (circle).

with optical power transmission provides a potential method for the recycle of optical pump after distributed amplification.

\section{CONCLUSION}

We have demonstrated an optically powered WDM communication system with distributed amplification in DSF and SMF. Clear and widely open eye diagrams have been obtained for all WDM channels during the experiment. BER plots for different channels during the DPA and DRA were measured with favorable results at the presence of 10-dB gain. Furthermore, in regards to the pump power requirement, when DSF is used as the transmission media for the distributed amplification, DPA, with lower pump power requirement, is the better candidate. But in the SMF, limited by the zero-dispersion wavelength, DRA becomes the only choice for C-band signal and DPA could still find its potential position for applications around $1300 \mathrm{~nm}$. Moreover, by recycling the residual optical pump power at the fiber output as the power supply for the receiving component, the energy-efficiency of the whole system has been realized. With photovoltaic cell of high conversion-efficiency and proper path-loss control, in addition to the 'last mile' transmission, our scheme could find potential application in the optically powered sensor networks.

\section{REFERENCES}

[1] B. C. DeLoach, "Sound alerter powered over an optical fiber," Bell Syst. Tech. J., vol. 53, pp. 3309-3316, 1978.

[2] R. C. Miller and B. C. DeLoach, "Optically powered speech communication over a fiber lightguide," Bell Syst. Tech. J., vol. 58, pp. 1735-1741, 1979.

[3] H. Kirkham and A. R. Johnston, "Optically powered data link for power system applications," IEEE Trans. Power Delivery, vol. 4, no. 4, pp. 1997-2004, Apr. 1989.

[4] S. J. Pemper, "A multiplexed network of optically powered, addressed and interrogated hybrid resonant sensors," Sens. Actuators A, vol. 47, pp. 474-477, 1978. 
[5] T. C. Banwell, R. C. Estes, L. A. Reith, Jr., P. W. Shumate, and E. M. Vogel, "Powering the fiber loop optically-A cost analysis," J. Lightw. Technol., vol. 11, no. 3, pp. 481-494, \Mar. 1993.

[6] R. Pena, C. Algora, I. R. Matłas, and M. Ĺpez-Amo, "Fiber-based 205-mW (27\% efficiency) power-delivery system for an all-fiber network with optoelectronic sensor units," Appl. Opt., vol. 38, no. 12, pp. 2463-2466, 1999.

[7] D. E. A. Clarke and T. Kanada, "Broadband: The last mile," IEEE Commun. Mag., vol. 31, no. 3, pp. 94-100, 1993.

[8] Jr. P. E. Green, "Paving the last mile with glass," IEEE Spectrum, vol. 39 , no. 12 , pp. 13-14, 2002.

[9] E. Leitgeb, J. Bregenzer, P. Fasser, and M. Gebhart, "Free space optics-Extension to fiber-networks for the "last mile"," in Proc. IEEE 15th LEOS, 2002, vol. 2, pp. 459-460.

[10] E. Leitgeb, M. Loschnigg, U. Birnbacher, G. Schwarz, and A. Merdonig, "High reliable optical wireless links for the last mile access," in Proc. 10th Anniv. Int. Conf. Transparent Opt. Netw., 2008, vol. 4, pp. 178-183.

[11] I. Nasieva, J. D. Ania-Castanon, and S. K. Turitsyn, "Nonlinearity management in fibre links with distributed amplification," IEEE Electron. Lett., vol. 39, no. 11, pp. 856-857, 2003.

[12] M. Vasilyev, "Raman-assisted transmission: Toward ideal distributed amplification," in Proc. Opt. Fiber Commun. Conf., 2003, vol. 1, pp. 303-305.

[13] C. McKinstrie, M. Yu, M. G. Raymer, and S. Radic, "Quantum noise properties of parametric processes," Opt. Exp., vol. 13, no. 13, pp. 4986-5012, 2005.

[14] M. Vasilyev, "Distributed phase-sensitive amplification," Opt. Exp., vol. 13, no. 19, pp. 7563-7571, 2005.

[15] D. Wake, A. Nkansah, N. J. Gomes, C. Lethien, C. Sion, and J. P. Vilcot, "Optically powered remote units for radio-over-fiber systems," J. Lightw. Technol., vol. 26, no. 15, pp. 2484-2491, 2008.

[16] H. Miyakawa, Y. Tanaka, and T. Kurokawa, "Design approaches to power-over-optical local-area-network systems," Appl. Opt., vol. 43, no. 6, pp. 1379-1389, 2004

[17] G. Bottger, M. Dreschmann, C. Klamouris, M. Hubner, M. Rger, A. W. Bett, T. Kueng, J. Becker, W. Freude, and J. Leuthold, "An optically powered video camera link," IEEE Photon. Technol. Lett., vol. 20, no. 1, pp. 39-41, 2008.

[18] G. Kalogerakis, M. E. Marhic, K. K. Y. Wong, and L. G. Kazovsky, "Transmission of optical communication signals by distributed parametric amplification," J. Lightw. Technol., vol. 23, no. 10, pp. 2945-2953, 2005.

[19] X. Xu, K. K. Y. Cheung, S. Yang, Y. Liang, T. I. Yuk, and K. K. Y. Wong, "Optically powered WDM signal transmission system with distributed parametric amplification," IEEE Photon. Technol. Lett., vol. 22, no. 16, pp. 1232-1234, 2010.

[20] N. Henmi, Y. Aoki, T. Ogata, T. Saito, and S. Nakaya, "A new design arrangement of transmission fiber dispersion for suppressing nonlinear degradation in long-distance optical transmission systems with optical repeater amplifiers," J. Lightw. Technol., vol. 11, no. 10, pp. 1615-1621, Nov. 1993.
[21] G. Bosco, A. Carena, V. Curri, R. Gaudino, P. Poggiolini, and S. Benedetto, "A novel analytical approach to the evaluation of the impact of fiber parametric gain on the bit error rate," IEEE Trans. Commun., vol. 49, no. 12, pp. 2154-2163, Dec. 2001.

[22] J. M. C. Boggio, S. Tenenbaum, and H. L. Fragnito, "Amplification of broadband noise pumped by two lasers in optical fibers," J. Opt. Soc. Amer. B, vol. 18, no. 10, pp. 1428-1435, 2001.

[23] P. Serena, A. Orlandini, and A. Bononi, "Parametric-gain approach to the analysis of single-channel DPSK/DQPSK systems with nonlinear phase noise," J. Lightw. Technol., vol. 24, no. 6, pp. 2026-2037, Jun. 2006.

[24] M. E. Marhic, Fiber Optical Parametric Amplifiers, Oscillators and Related Devices. Cambridge, U.K.: Cambridge Univ. Press, 2007.

[25] J. Hansryd, P. A. Andrekson, M. Westlund, J. Li, and P. O. Hedekvist, "Fiber-based optical parametric amplifiers and their applications," IEEE J. Sel. Topics Quantum Electron., vol. 8, no. 3, pp. 506-520, May/Jun. 2002.

[26] S. Namiki, S. Koji, N. Tsukiji, and S. Shikii, "Challenges of Raman amplification," Proc. IEEE, vol. 94, no. 5, pp. 1024-1035, 2006.

[27] J. Bromage, "Raman amplification for fiber communications systems," J. Lightw. Technol., vol. 22, no. 1, pp. 79-93, 2004.

[28] C. Headley and G. P. Agrawal, Raman Amplification in Fiber Optical Communication Systems. New York: Academic, 2005.

[29] H. Miyakawa, Y. Tanaka, and T. Kurokawa, "Optical power and signal transmission with WDM single mode fiber optic systems," in Proc. Renewable Energy, 2006, pp. 317-320.

[30] T. Torounidis, H. Sunnerud, P. O. Hedekvist, and P. A. Andrekson, "Amplification of WDM signals in fiber-based optical parametric amplifiers," IEEE Photon. Technol. Lett., vol. 15, no. 8, pp. 1061-1063, Aug. 2003.

[31] C. R. S. Fludger, V. Handerek, and R. J. Mears, "Pump to signal RIN transfer in Raman fiber amplifiers," J. Lightw. Technol., vol. 19, no. 8, pp. 1061-1063, Aug. 2003.

[32] S. Namiki, S. Koji, N. Tsukiji, and S. Shikii, "Challenges of Raman amplification," Proc. IEEE, vol. 94, no. 5, pp. 1024-1035, 2006.

[33] J. Bromage, "Raman amplification for fiber communications systems," J. Lightw. Technol., vol. 22, no. 1, pp. 79-93, Jan. 2004.

[34] V. Andreev, V. Khvostikov, V. Kalinovsky, V. Lantratov, V. Grilikhes, V. Rumyantsev, M. Shvarts, M. Fokanov, and A. Pavlov, "High current density GaAs and GaSb photovoltaic cells for laser power beaming," in Proc. 3rd World Conf. PV Energy Conv., 2003, vol. 1, pp. 761-764.

[35] D. Krut, R. Sudharsanan, T. Isshiki, R. King, and N. H. Karam, "A 53\% high efficiency GaAs vertically integrated multi-junction laser power converter," in Proc. Device Res. Conf., 2007, vol. 1, pp. 123-124.

Author biographies not included at authors' request due to space constraints. 